Waves 8 Oscillations in the Solar Atmosphere:

Heating and Magneto-Seismology

Proceedings IAU Symposium No. 247, 2007

R. Erdélyi $\&$ C. A. Mendoza-Briceño, eds.

(C) 2008 International Astronomical Union doi:10.1017/S1743921308014609

\title{
Progress in coronal seismology
}

\author{
B. Roberts \\ Mathematical Institute, University of St Andrews, St Andrews, Fife KY16 9SS, Scotland, UK \\ email: bernie@mcs.st-and.ac.uk
}

\begin{abstract}
Coronal seismology is now a well developed area of solar physics, even though many questions remain for resolution. Here we take stock of the progress made since the first direct imaging of oscillating loops was achieved through TRACE spacecraft observations in 1999.
\end{abstract}

Keywords. Sun: oscillations, MHD waves, coronal seismology

\section{Introduction}

Coronal seismology is a marriage of theory and observations with the aim of producing a detailed knowledge of physical parameters in the corona. It aims to estimate such quantities as the magnetic field strength in a coronal loop, the width of the loop and the steepness of its density or magnetic field profile across the loop, or the longitudinal density scale height along the loop. Also, it offers the prospect of determining the thermal, viscous or ohmic damping coefficients of the coronal plasma. All these quantities are difficult to obtain by direct measurement, so coronal seismology offers an important way of progressing in their determination. The subject exploits observations of coronal oscillations by matching such observations to predicted theoretical results derived for a model loop. The same ideas may be applied elsewhere in the solar atmosphere, including the photosphere or chromosphere and in prominences, and indeed in stellar atmospheres.

The seismology we describe is based upon a magnetohydrodynamic description of the plasma. So, although there are natural similarities with helioseismology there are also significant differences: helioseismology is in the main built upon the behaviour of a single wave, the sound wave, whereas magnetohydrodynamic (MHD) seismology in principle draws on the properties of three waves - the slow and fast magnetoacoustic waves and the Alfvén wave.

It is of interest to examine how coronal seismology began. Uchida (1970), exploring theoretically the behaviour of fast waves in a complex magnetic field for the purpose of explaining observed Moreton waves, suggested that when combined with a knowledge of the density distribution in a stratified corona this work could be exploited to obtain a "seismological diagnosis" of the distribution of magnetic field in the corona. This provides a global estimate of a mean coronal atmosphere (see also Uchida 1968, 1973, 1974). A recent discussion of global coronal seismology is given in Ballai, Erdélyi \& Pintér (2005).

The suggestion that oscillations could be used as a means of local coronal seismology was made in Roberts, Edwin \& Benz (1984), independently of the work of Uchida. Exploiting the theory of oscillations of a magnetic flux tube embedded in a magnetised atmosphere (Edwin \& Roberts 1983), Roberts, Edwin \& Benz (1984) suggested that "magnetoacoustic oscillations provide a potentially useful diagnostic tool for determining physical conditions in the inhomogeneous corona". The authors commented that the combination of theory and observations provides "a valuable diagnostic tool for in situ conditions in the corona", allowing determinations of the local Alfvén speed and spatial dimension of the coronal inhomogeneity that forms a loop. 
The discovery in 1999 of directly EUV-imaged oscillations in coronal loops detected by the spacecraft TRACE (Aschwanden et al. 1999; Nakariakov et al. 1999), gave a major impetus to this field, since it moved observations and theory together and towards an unambiguous identification of the oscillations (see also Aschwanden et al. 2002; Schrijver et al. 1999; Schrijver, Aschwanden \& Title 2002). Added to which, quite different oscillations were shortly thereafter detected by the ground-based instrument SECIS (Williams et al. 2001, 2002a; see also Katsiyannis et al. 2003) and by the spacecrafts SoHO (Wang et al. 2002, 2003a, b; see also Kliem et al. 2002), Yohkoh (Mariska 2005, 2006) and RHESSI (Foullon et al. 2005). Taken together with the long history of spatially unresolved radio observations of oscillatory phenomena in the corona (see Aschwanden 1987), and the detection of oscillations in coronal plumes (Ofman et al. 1997; DeForest \& Gurman 1998) and also in prominences (Oliver \& Ballester 2002; Ballester 2003), we see the development of a rich subject - coronal seismology.

It is interesting to note that applications of coronal seismology are spreading; by coronal seismology, we here mean the technique of combining observations with magnetohydrodynamic wave theory, which of course can also be applied outside the corona (with perhaps only minor modifications). In this regard it is interesting to note the new applications to spicules (Kukhianidze, Zaqarashvili \& Khutsishvili 2006; Singh \& Dwivedi 2007; Zaqarashvili et al. 2007) and indeed to loops in stellar coronae (Mitra-Kraev et al. 2005; Mathioudakis et al. 2006).

Following the direct imaging of oscillating loops by TRACE, the theoretical framework introduced through the dispersion diagram of Edwin \& Roberts (1983) was used as a means of interpreting the observations (Roberts et al. 1984); the topic was reviewed in Roberts (2000), where also various issues then judged problematic were outlined. Here we take stock of the considerable progress made in the field since the observational discoveries of 1999. Reviews of theoretical aspects are also given in Roberts (2002, 2004), Roberts \& Nakariakov (2003), Goossens et al. (2005) and Goossens, Andries \& Arregui (2006). Observations have been reviewed in Wang (2004, 2006) and Banerjee et al. (2007). Broad overviews are given in Aschwanden (2004), Nakariakov \& Verwichte (2005), De Moortel (2005) and Nakariakov (2007).

\section{Basics}

The discussion of the modes of oscillation of a magnetic flux tube is generally based upon the simple configuration of a straight cylindrical tube embedded in a uniform magnetic atmosphere; the tube is defined by virtue of differences in magnetic field strength, plasma density and temperature within a cylinder of radius $a$ and conditions in the uniform magnetised surroundings. The plasma density $\rho_{0}$ inside the tube and the density $\rho_{e}$ outside the tube are particularly important. Inside the tube the sound speed is $c_{s}$ and the Alfvén speed is $c_{A}$, with an associated slow speed $c_{t}$ given by (Defouw 1976; Roberts \& Webb 1978)

$$
c_{t}=c_{s} c_{A} /\left(c_{s}^{2}+c_{A}^{2}\right)^{1 / 2} .
$$

In the environment of the tube, the respective sound, Alfvén and slow speeds are $c_{s e}$, $c_{A e}$ and $c_{t e}$. Magnetostatic pressure balance coupled with the ideal gas law requires that the plasma densities $\rho_{0}$ and $\rho_{e}$ are related to the propagation speeds by

$$
\frac{\rho_{e}}{\rho_{0}}=\frac{c_{s}^{2}+\frac{1}{2} \gamma c_{A}^{2}}{c_{s e}^{2}+\frac{1}{2} \gamma c_{A e}^{2}},
$$

where $\gamma$ is the ratio of specific heats (generally taken to be $\gamma=5 / 3$ ). 
The linearised equations of magnetohydrodynamics for such a configuration lead to the dispersion relation

$$
\frac{1}{\rho_{0}\left(k_{z}^{2} c_{A}^{2}-\omega^{2}\right)} n_{0} \frac{J_{m}^{\prime}\left(n_{0} a\right)}{J_{m}\left(n_{0} a\right)}=\frac{1}{\rho_{e}\left(k_{z}^{2} c_{A e}^{2}-\omega^{2}\right)} m_{e} \frac{K_{m}^{\prime}\left(m_{e} a\right)}{K_{m}\left(m_{e} a\right)} .
$$

Equation (2.3) determines the propagation speed $c\left(=\omega / k_{z}\right)$ of a wave along the tube; this relationship, connecting the speed $c$ (or the frequency $\omega$ ) and the longitudinal wavenumber $k_{z}$, is transcendental and, except in some limiting cases, has to be investigated numerically (Edwin \& Roberts 1983). The pressure or velocity perturbations are given by the linearised MHD wave equations coupled with the solution of (2.3). Specifically, inside the tube $(r<a)$ variations in total pressure $p_{T}$ are of the form

$$
p_{T}(r, \theta, z, t)=A_{0} J_{m}\left(m_{0} r\right) \operatorname{expi}\left(\omega t-m \theta-k_{z} z\right)
$$

where $A_{0}$ is an arbitrary constant. The mode number $m(=0,1,2, \ldots)$ describes geometrically the shape of the oscillating tube: sausage modes occur when $m=0$, kink modes when $m=1$, and fluting modes when $m \geqslant 2$. Dispersion relation (2.3) has been discussed in detail in Edwin \& Roberts (1983). Aspects of (2.3), or some of its special cases, have been discussed in, for example, McKenzie (1970), Zaitsev \& Stepanov (1975), Ryutov \& Ryutova (1976), Roberts \& Webb (1978), Spruit (1982) and Cally (1986). Here $J_{m}(r)$ and $K_{m}(r)$ denote Bessel and modified Bessel functions with derivatives $J_{m}^{\prime}(r)$ and $K_{m}^{\prime}(r)$ (Abramowitz \& Stegun 1965); in the dispersion relation (2.3) these terms are calculated at $n_{0} a$ or $m_{e} a$ (as appropriate), where

$$
n_{0}^{2}=-\frac{\left(k_{z}^{2} c_{s}^{2}-\omega^{2}\right)\left(k_{z}^{2} c_{A}^{2}-\omega^{2}\right)}{\left(c_{s}^{2}+c_{A}^{2}\right)\left(k_{z}^{2} c_{t}^{2}-\omega^{2}\right)}, \quad m_{e}^{2}=\frac{\left(k_{z}^{2} c_{s e}^{2}-\omega^{2}\right)\left(k_{z}^{2} c_{A e}^{2}-\omega^{2}\right)}{\left(c_{s e}^{2}+c_{A e}^{2}\right)\left(k_{z}^{2} c_{t e}^{2}-\omega^{2}\right)} .
$$

The dispersion relation (2.3) describes the magnetoacoustic waves of a tube, having $p_{T} \neq 0$. It is also possible to have Alfvén waves, which propagate with either the speed $c_{A}$ or $c_{A e}$, and correspond to torsional oscillations of the tube. Such modes are difficult to detect observationally (Zaqarashvili 2003).

The dispersion relation (2.3) is subject to the requirement that $m_{e}>0$, ensuring that disturbances in the environment decay far from the tube (as $r \rightarrow \infty)$. The restriction $m_{e}>0$ imposed on (2.3) means that far from the tube there is no appreciable disturbance: waves are essentially confined to the tube. However, the dispersion relation is valid whatever the nature of $n_{0}$; inside the tube (for $r<a$ ), disturbance may be oscillatory or non-oscillatory (exponential). Modes that are oscillatory inside the tube are termed body waves $\left(n_{0}^{2}>0\right)$; modes that are non-oscillatory inside the tube are surface waves $\left(n_{0}^{2}<0\right)$ (see Roberts 1981a, b; Edwin \& Roberts 1982, 1983).

It turns out that under coronal conditions, in which $c_{A}>c_{s}$ and $c_{A e}>c_{s e}$, two sets of body waves arise, the slow and fast body waves, with no surface waves (Edwin \& Roberts 1983). These are best displayed graphically. Figure 1 shows the results. We see a group of slow waves well separated from a group of fast waves. The slow waves are only mildly dispersive, giving

$$
\omega \approx k_{z} c_{t}, \quad c \approx c_{t},
$$

whether the wave is a sausage mode or a kink mode. By contrast, the fast waves are more strongly dispersive with speeds $c$ of propagation along the tube depending precisely on the wavenumber $k_{z}$, but in any case lying between the two Alfvén speeds. In fact, these waves arise only if $c_{A e}>c_{A}$ and then the fast waves (be it the sausage mode or the kink mode) have speeds $c$ that lie in:

$$
c_{A}<c<c_{A e}
$$




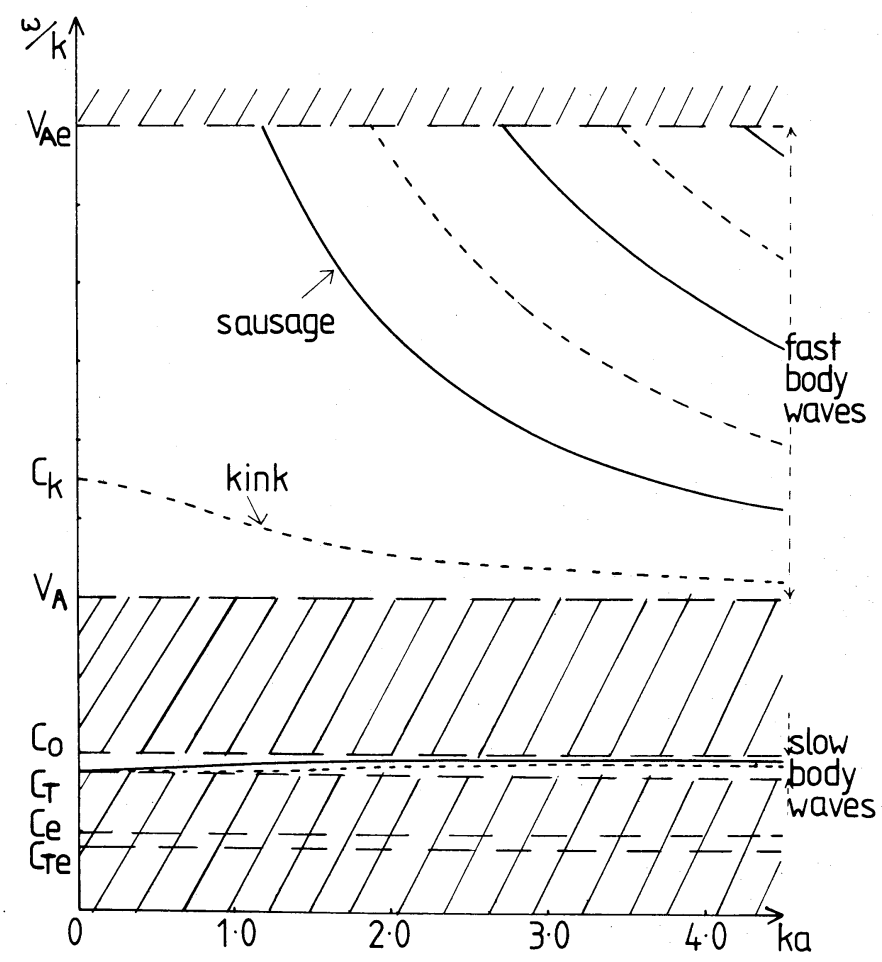

Figure 1. The dispersion diagram of Edwin \& Roberts (1983), showing solutions of the dispersion relation (2.3) for a magnetic flux tube under coronal conditions, namely $c_{s}<c_{A}<c_{A e}$. Specifically, $c_{A e}=5 c_{s}, c_{s e}=c_{s} / 2$, and $c_{A}=2 c_{s}$; these values give $\rho_{0} \approx 4.9 \rho_{e}, c_{t} \approx 0.89 c_{s}$ and $c_{k} \approx 1.38 c_{A} \approx 0.55 c_{A e}$. The diagram gives the phase speed $c\left(=\omega / k_{z}\right)$ as a function of the dimensionless wavenumber $k_{z} a$ for fast and slow magnetoacoustic body modes in a coronal magnetic flux tube of radius $a$. [Note the notational changes from the present text: $V_{A} \equiv c_{A}$, $V_{A e} \equiv c_{A e}, C_{0} \equiv c_{s}, C_{T} \equiv c_{t}, C_{T e} \equiv c_{t e}, C_{e} \equiv c_{s e}$, and $\left.k \equiv k_{z}.\right]$

In the low $\beta$ plasma of the typical corona the condition $c_{A e}>c_{A}$ corresponds to $\rho_{0}>\rho_{e}$, applying to a dense tube.

Of particular interest is the occurrence of the fast kink mode that in the limit of a thin tube, $k_{z} a \ll 1$, gives a mode which propagates with the speed $c_{k}$ (see figure 1 ). Specifically, the principal kink mode gives (Ryutov \& Ryutova 1976; Parker 1979; Spruit 1982; Edwin \& Roberts 1983)

$$
\omega \approx k_{z} c_{k}, \quad c \approx c_{k}
$$

where

$$
c_{k}=\left(\frac{\rho_{0} c_{A}^{2}+\rho_{e} c_{A e}^{2}}{\rho_{0}+\rho_{e}}\right)^{1 / 2}
$$

defines the kink speed $c_{k}$ in terms of the Alfvén speeds inside and outside the tube and the respective plasma densities $\rho_{0}$ and $\rho_{e}$, related by equilibrium pressure balance (2.2). The speed $c_{k}$ is intermediate between $c_{A}$ and $c_{A e}$. In the particular case of an everywhere uniform magnetic field - so that the tube is defined in terms of density or temperature (but not pressure) contrast - then

$$
c_{k}=\left(\frac{2 \rho_{0}}{\rho_{0}+\rho_{e}}\right)^{1 / 2} c_{A} .
$$


For the extreme of a high density tube, $\rho_{0} \gg \rho_{e}, c_{k}=\sqrt{2} c_{A}$; thus, the kink speed in a dense coronal flux tube is some $41 \%$ higher than the Alfvén speed within the tube.

The principal kink mode exists for all $k_{z} a$, and the wave is moderately dispersive. This is in contrast to the other fast kink oscillations and also the fast sausage modes, shown in figure 1 , which exist only for sufficiently large $k_{z} a$; these waves have a cutoff. If $k_{z} a$ is too small, both the fast kink waves and the fast sausage waves become leaky (Cally 1986; see also Roberts \& Webb 1979). Of the fast waves, only the principal kink mode exists as a trapped mode of the flux tube, whatever the wavenumber $k_{z}$. This feature of wave cutoff has important implications for both propagating waves (Roberts, Edwin \& Benz 1983; Roberts et al. 1984; Pascoe, Nakariakov \& Arber 2007) and standing waves (Roberts et al. 1984; Nakariakov, Melnikov \& Reznikova 2003). If $c_{A}>c_{A e}$, then the fast wave is no longer a trapped mode of the tube.

Finally, we note that observations have so far detected a substantial proportion of figure 1 , though concentrating mainly on $k_{z} a \ll 1$, the long wavelength limit of a thin loop. For impulsively excited waves all wavenumbers are important and so the fast mode cutoffs that are apparent in figure 1 play a role, as they do for waves in tubes that are not thin. Observations are now making progress in this direction too.

\section{Coronal seismology results}

We turn now to an application of the dispersion diagram to observations. We consider results that depend directly upon the simple model of a magnetic flux tube that gave rise to figure 1 , leaving aside various complexities until $\S 4$.

\subsection{Fast kink mode: standing oscillations}

Consider the principal fast kink wave; as a standing oscillation, this is a global vibration of the tube as a whole. Such global disturbances were the first to be directly imaged by TRACE (Aschwanden et al. 1999, 2002; Nakariakov et al. 1999; Schrijver et al. 2002; Wang \& Solanki 2004). As noted above, for a thin coronal loop the principal fast kink mode propagates with a speed $c_{k}$ given by $(2.9)$, and this may be related to the period $P(=2 \pi / \omega)$ through the loop length once the wavenumber $k_{z}$ is specified. We consider the application of (2.8) to standing waves in a loop of length $\dagger 2 L$. Then line-tying of the magnetic field lines in the dense lower atmosphere (photosphere-chromosphere) ensures that any disturbance in the corona is unable to perturb the ends of the loop. Accordingly, we take

$$
k_{z}=N \pi /(2 L)
$$

with the integer $N$ describing the oscillations along the loop: $N=1$ gives the fundamental mode (which disturbs globally the whole of the loop, save its anchored ends); $N=2$ gives the first harmonic of the fundamental. The period of each of these oscillations is given by the relations (2.8) and (3.1). For the fundamental global mode, we have

$$
P_{1}=P_{\text {kink }} \equiv \frac{4 L}{c_{k}}=\frac{2 \times \text { loop length }}{\text { mode speed }} .
$$

When coupled with observations, (3.2) may be used directly to determine $c_{k}$ (and thus $c_{A}$, once assumptions are made about the density enhancement and field strengths $B_{0}$ and $B_{e}$ are made); this was done in Roberts et al. (1984), using the then available data. Alternatively, following Nakariakov \& Ofman (2001), (3.2) may be rearranged to give

$\dagger$ Care is needed here in reading the literature, since some authors use $L$ for loop length whilst others use $2 L$. 
the magnetic field strength explicitly. Assuming for simplicity that the magnetic field is everywhere uniform, of strength $B_{0}$ both inside and outside the loop, then

$$
B_{0}=\left(\mu \rho_{0}\right)^{1 / 2} c_{A}=\sqrt{2 \mu} \frac{2 L}{P_{\text {kink }}} \rho_{0}{ }^{1 / 2}\left(1+\rho_{e} / \rho_{0}\right)^{1 / 2} .
$$

Equation (3.3) gives the loop field strength in mks units, with $B_{0}$ in tesla.

Nakariakov \& Ofman (2001) used the equivalent $\dagger$ of (3.3) to determine $B_{0}$ for a TRACE loop. Verwichte et al. (2004) have further applied (3.3) to observations of nine oscillating TRACE loops.

\subsection{Fast kink mode: propagating waves}

In contrast to the standing waves, there has been far less observational information about propagating kink waves. Nonetheless, there have been some observations which seem have a natural interpretation in terms of propagating waves. Propagating transverse waves have been detected in an open magnetic field structure by $T R A C E$. Verwichte, Nakariakov \& Cooper (2005) report dark 'tadpole'-like structures moving downward in a post-flare arcade. Quasi-periodic transverse displacements of dark tadpole tails are seen, with periods in the range of $90-220 \mathrm{~s}$. Phase speeds are in the range $200-700 \mathrm{~km} \mathrm{~s}^{-1}$ at a height of $90 \mathrm{Mm}$ above the arcade, falling to $90-200 \mathrm{~km} \mathrm{~s}^{-1}$ at a height of $60 \mathrm{Mm}$. Verwichte, Nakariakov \& Cooper (2005) interpreted the phenomenon as propagating fast magnetoacoustic kink waves guided in a vertical and evolving open magnetic structure.

\subsection{Fast sausage mode: standing oscillations}

As noted earlier, figure 1 shows that the sausage modes - distinct from the principal kink mode - possess a low wavenumber cutoff. For the fast sausage mode to be a trapped mode of the tube it is necessary that $k_{z}>k_{z}^{\text {cutoff }}$, where the cutoff wavenumber $k_{z}^{\text {cutoff }}$ is given by (Edwin \& Roberts 1983: Roberts et al. 1984)

$$
k_{z}^{\text {cutoff }}=\frac{j_{0}}{a}\left[\frac{\left(c_{s}^{2}+c_{A}^{2}\right)\left(c_{A e}^{2}-c_{t}^{2}\right)}{\left(c_{A e}^{2}-c_{A}^{2}\right)\left(c_{A e}^{2}-c_{s}^{2}\right)}\right]^{1 / 2},
$$

where $j_{0}$ denotes the zeros of the Bessel function $J_{0}$; cutoff arises when $\omega=k_{z} c_{A e}$, $c=c_{A e}$. There are an infinite number of zeros of the function $J_{0}$; the first zero has $j_{0}=2.4048$. In the limit of a high external Alfvén speed $\left(c_{A e} \gg c_{s}, c_{t}\right),(3.4)$ reduces to

$$
k_{z}^{\text {cutoff }}=\frac{j_{0}}{a}\left(\frac{c_{s}^{2}+c_{A}^{2}}{c_{A e}^{2}-c_{A}^{2}}\right)^{1 / 2} .
$$

If further the plasma inside the tube is low $\beta$, so that $c_{A} \gg c_{s}$ (and pressure balance in the equilibrium configuration is achieved by requiring that the magnetic field is everywhere uniform, the tube being essentially defined as a region of plasma density difference, $\rho_{0} \neq \rho_{e}$, with $\rho_{0} c_{A}^{2}=\rho_{e} c_{A e}^{2}$ ), then the cutoff expression reduces to (Roberts et al. 1984; Nakariakov, Melnikov \& Reznikova 2003; Aschwanden, Nakariakov \& Melnikov 2004)

$$
k_{z}^{\text {cutoff }}=\frac{j_{0}}{a}\left(\frac{c_{A}^{2}}{c_{A e}^{2}-c_{A}^{2}}\right)^{1 / 2}=\frac{j_{0}}{a}\left(\frac{\rho_{e}}{\rho_{0}-\rho_{e}}\right)^{1 / 2} .
$$

Accordingly, with $k_{z}$ quantized (by (3.1)) to fit into a loop of length $2 L$, we may express the cutoff condition in terms of the loop half-length $L$ and tube radius $a$, yielding

$$
\frac{L}{a}<\frac{\pi N}{2 j_{0}}\left(\frac{c_{A e}^{2}}{c_{A}^{2}}-1\right)^{1 / 2} .
$$

$\dagger$ Note that in Nakariakov \& Ofman (2001), $2 L$ is there taken to be the height of the coronal loop, modelled as circular with the ends of the circle embedded in the lower atmosphere. 
In the zero $\beta$ plasma, taking the first zero for $j_{0}$ gives (Nakariakov et al. 2003)

$$
\frac{L}{a}<0.6532 N\left(\frac{\rho_{0}}{\rho_{e}}-1\right)^{1 / 2}, \quad \frac{a}{L}>\frac{1.5309}{N} \frac{1}{\left(\frac{\rho_{0}}{\rho_{e}}-1\right)^{1 / 2}} .
$$

It is apparent that the inequality favours dense $\left(\rho_{0} \gg \rho_{e}\right)$ and short, fat loops $(a / L$ of order unity) or high integer orders $N$.

It is of interest to note the period $P^{\text {cutoff }}\left(=2 \pi / \omega^{\text {cutoff }}\right)$ of the wave at the cutoff frequency $\omega=\omega^{\text {cutoff }}=k_{z}^{\text {cutoff }} c_{A e}$; for the zero $\beta$ plasma, this is

$$
P^{\text {cutoff }}=\frac{2 \pi a}{j_{0}}\left(\frac{1}{c_{A}^{2}}-\frac{1}{c_{A e}^{2}}\right)^{1 / 2}=\frac{2 \pi a}{j_{0} c_{A}}\left(\frac{\rho_{0}-\rho_{e}}{\rho_{0}}\right)^{1 / 2} .
$$

In the extreme of a high density loop, $\rho_{0} \gg \rho_{e}$, this gives $P^{\text {cutoff }}=2.6 a / c_{A}$.

The Nobeyama radioheliograph observation explored by Nakariakov et al. (2003; see also Aschwanden, Nakariakov \& Melnikov 2004; Reznikova et al. 2006) suggests a loop with internal particle number density of $10^{17} \mathrm{~m}^{-3}$ compared with an environment number density of $2 \times 10^{15} \mathrm{~m}^{-3}$, yielding a density ratio of $\rho_{0} / \rho_{e}=50$; thus $a / L>0.22 / N$. With a loop length of $25 \mathrm{Mm}$ (so that $L=12.5 \mathrm{Mm}$ ) this requires a tube radius of $a>2.73 \mathrm{Mm}$ (or width in excess of $5.47 \mathrm{Mm}$ ) for the $N=1$ mode. Determinations of the tube width suggest a tube diameter of $6 \mathrm{Mm}$, consistent with the principal sausage mode $(N=1)$ of the loop. For $c_{A}=480 \mathrm{~km} \mathrm{~s}^{-1}$, the period $P=P_{1}$ of the global oscillation is of order the cutoff period $2.62 a / c_{A}$, i.e. $P_{1} \approx 15 \mathrm{~s}$; this compares favourably with the observed period of $14-17 \mathrm{~s}$.

By contrast, in a typical TRACE loop with small $a / L$ and moderate density ratio $\rho_{0} / \rho_{e}$ it is evident that the fast sausage mode can arise as a trapped mode only for large values of the integer $N$; specifically, for $a / L=10^{-1}$ and a density ratio of $\rho_{0} / \rho_{e}=5$ we find that $N$ must be of order 8 , and for much thinner loops this requirement increases accordingly. This is the conclusion reached by Roberts et al. (1984) for typical coronal loops. Only in short, fat and very dense loops, as discussed by Nakariakov et al. (2003) for certain loops observed by the Nobeyama radioheliograph, can the fundamental $(N=1)$ global mode arise as a trapped sausage oscillation of the loop as a whole.

\subsection{Fast sausage mode: propagating waves}

The cutoff frequency also plays an important role in the behaviour of propagating fast waves. Indeed, the full form of the dispersion relation (2.3) comes into force when we consider impulsively excited waves, for then the whole spectrum of frequencies is involved in the determination of the generated wave. Impulsively excited fast sausage waves were discussed in some detail by Roberts et al. (1983, 1984), drawing on an analogy with sound waves in an ocean layer (Pekeris 1948). An impulsively excited wave exhibits a three phase temporal signal, consisting of a period phase, a quasi-period phase, and a decay phase. The cutoff frequency determines the largest period of oscillation carried by the propagating wave. It is interesting to note that Williams et al. (2001, 2002a) have observed the rapid propagation of an oscillatory disturbance in a loop. This remarkable result was achieved by the deployment of ground-based rapid photography using the camera instrument SECIS, taking 44 frames per second of the eclipsed corona. Comparisons with $\mathrm{SoHO}$ observations of the corona then allowed a loop to be identified as a candidate for supporting the oscillation. The loop, of length $200 \mathrm{Mm}$ and radius $a=5 \mathrm{Mm}$, supported a propagating wave travelling along the loop with a speed of $2100 \mathrm{~km} \mathrm{~s}^{-1}$ and carrying a periodicity of $6 \mathrm{~s}$. 
We can explain this observation in terms of the theory given by Roberts et al. (1983, 1984) for the fast sausage mode. Dispersion in the mode means that a wave packet, generated impulsively so that all frequencies are excited, propagates with the group velocity of the wave and carries an oscillation with a period of order $P^{\text {cutoff }}$ given by (3.9). Numerical simulations of this effect have been given in Nakariakov et al. (2004), Nakariakov, Pascoe \& Arber (2005) and Pascoe, Nakariakov \& Arber (2007a), using a smooth cross-field density profile (see Nakariakov \& Roberts 1995); Pascoe, Nakariakov \& Arber (2007b) has also discussed multi-shell structures. In fact, the timescale $P^{\text {cutoff }}$ is the longest timescale in the impulsively excited wave packet (Roberts et al. 1984). The densities reported by Williams et al. give a ratio $\rho_{e} / \rho_{0}=0.4$. Following Roberts (2004), we associate the observed speed of $2100 \mathrm{~km} \mathrm{~s}^{-1}$ with the group velocity $c_{g}$ of a fast wave, determined numerically from the dispersion relation (2.3). Then, taking $P^{\text {cutoff }}=6 \mathrm{~s}$ and $\rho_{e} / \rho_{0}=0.4$, equation (3.9) implies a tube radius of $a=6216\left(c_{A} / c_{g}\right) \mathrm{km}$. So with $c_{g} \approx c_{A}$ we obtain a tube radius of approximately $6000 \mathrm{~km}$ (and probably smaller than this estimate, since $P^{\text {cutoff }}$ is an upper limit to the timescale in the quasi-periodic phase of the magnetic Pekeris wave). This determination of radius is entirely consistent with the observationally determined radius of $5000 \mathrm{~km}$ (Williams et al. 2002b). Alternatively, we could have used the oscillations together with the observationally determined tube radius to determine the density ratio $\rho_{e} / \rho_{0}$.

\subsection{Slow modes: standing oscillations}

The discovery of acoustic-like longitudinal oscillations in hot flare loops (Wang et al. 2002, 2003a, b) raises their interpretation in terms of slow mode waves. Consider (2.6) for a slow mode standing in a loop of length $2 L$; expressed in terms of the plasma beta $\beta$ inside the loop, the period of slow standing modes is

$$
P_{\text {slow }}=\frac{4 L}{c_{t}}=\frac{4 L}{c_{s}}\left(1+\frac{1}{2} \gamma \beta\right)^{1 / 2} .
$$

Wang et al. (2003a, b) report an oscillation on 15 April 2002 that had a period of 17.6 minutes in a loop of length $2 L=191 \mathrm{Mm}$. According to (3.10), this implies a slow speed of $c_{t}=362 \mathrm{~km} \mathrm{~s}^{-1}$. At a temperature of $6.3 \times 10^{6} \mathrm{~K}$ the sound speed is $c_{s}=417 \mathrm{~km} \mathrm{~s}^{-1}$, implying an Alfvén speed of $729 \mathrm{~km} \mathrm{~s}^{-1}$ and a plasma beta of $\beta=0.39$. An Alfvén speed of $729 \mathrm{~km} \mathrm{~s}^{-1}$ implies a magnetic field strength of $B_{0}=24 \mathrm{G}$ for a number density of $5 \times 10^{15} \mathrm{~m}^{-3}$. Recently, Wang, Innes \& Qiu (2007) have applied this approach to the analysis of seven $S U M E R$ Doppler events, finding that $\beta$ is in the range $0.15-0.91$, with a magnetic field strength in the range $12-61 \mathrm{G}$.

\subsection{Slow modes: propagating waves}

Propagating longitudinal waves have been detected in the legs of loops (Berghmans \& Clette 1999; De Moortel, Ireland \& Walsh 2000; Robbrecht et al. 2001; De Moortel et al. 2002a-c) and seem to have a natural interpretation in terms of the slow mode (Ofman, Nakariakov \& DeForest 1999; Nakariakov, Ofman \& Arber 2000). Figure 1 shows that under coronal conditions the slow mode has only a weak dispersion, and propagates sub-sonically with the tube speed $c_{t}$. Under typical TRACE loop conditions this gives a speed only very slightly below the sound speed inside the tube; for example, a sound speed of $c_{s}=200 \mathrm{~km} \mathrm{~s}^{-1}$ and an Alfvén speed of $c_{A}=1000 \mathrm{~km} \mathrm{~s}^{-1}$ produce a tube speed of $c_{t}=196 \mathrm{~km} \mathrm{~s}^{-1}$. The observed waves penetrate only some $10 \%$ into the loop before they are lost from view and are presumably damped. Thermal conduction seems to be an efficient mechanism of damping (see $\S 4.2$ ). 


\section{Complexity}

So far in our discussion we have focussed on an interpretation of the basic modes of a coronal loop modelled as a straight, uniform, magnetic flux tube embedded in a uniform magnetised environment. But it is natural to ask how these ideas are modified when we depart from this simple state and consider complications of structure, geometry, and physics. In order for our model to be useful it must also be robust, i.e., deductions made from a simple model must not be destroyed by modifications, though of course changes in the results will arise. Indeed, such changes may by themselves form a new arm for coronal seismology. Here we discuss briefly such aspects.

The cross-sectional shape of the basic flux tube is of interest since there is no compelling reason to assume it must always be circular in cross-section. Ruderman (2003) has recently investigated the modes of oscillation of a flux tube with elliptical crosssection, assuming the tube to be in a $\beta=0$ plasma. He finds that there are two kink modes of oscillation, reflecting the possibility for the tube to move either along the major axis or the minor axis of the ellipse. In the extreme of a highly elongated elliptical crosssection, the modes of oscillation of a magnetic slab (Edwin \& Roberts 1982) are recovered (Ruderman 2003). In general, though, we can conclude that ellipticity is not a major effect in our modelling of observed coronal loop oscillations.

The curvature of a magnetic loop is another geometrical effect that might be expected to have interesting consequences for our understanding of loop oscillations, but a study by Van Doorsselaere et al. (2004a) suggests that such effects are of limited importance, though curvature does introduce some leakage of the modes.

There is ever reason to consider twist in a flux tube, since many observations of loops indicate such a possibility. Twist in an incompressible tube has been considered by Bennett, Roberts \& Narain (1999), following on from Dungey \& Loughhead (1954). This work has recently been extended by Erdélyi \& Carter (2006), Carter \& Erdélyi (2007), Erdélyi \& Fedun $(2006,2007)$ and Ruderman (2007). However, although the effects of twist are interesting they seem unlikely to lead to any major revision in our understanding of the modes on oscillation of a coronal flux tube.

The assumptions of uniformity within and external to the tube are of obvious concern. Indeed, allowing for radial variation in the equilibrium plasma density or magnetic field leads to resonant absorption (e.g., Goossens 1991), and thus to the appearance of damping in an otherwise ideal medium. In any case radial variation has consequences for the modes of oscillation (Solovev et al. 2002a, b; Kopylova, Stepanov \& Tsap 2002; Mikhalyaev 2005) and their damping. There are reasons to believe that loops possess a fine-scale structure but the nature of the fibril loop remains uncertain. Nonetheless, it is important to assess the effects of fine-scale structuring (Díaz, Oliver \& Ballester 2005; Ofman 2005; Luna et al. 2006; Gruszecki et al. 2006; Arregui et al. 2007b).

As first suggested by Díaz (2004), longitudinal structuring is also of considerable interest, since it can be expected to arise from a variety of effects including heating and stratification; longitudinal structuring can be analysed in terms of the period ratio between the fundamental mode and its harmonics.

Flows are also known to occur in coronal flux tubes, and may indeed give greater definition to the appearance of a coronal tube. Modelling flows in coronal tubes, be they in the form of draining flows, or filling upflows, or generally non-steady, is problematic and only a start on this work has so far been possible. The simplest case to consider is the presence of steady flows (either within a coronal flux tube or in its environment); both magnetic slabs (Nakariakov \& Roberts 1995a; Joarder, Nakarikov \& Roberts 1997 Tirry et al. 1998) and magnetic tubes (Uberoi \& Somasundaram 1980; Narayanan 1991; 
Somasundaram, Venkatraman \& Sengottuvel 1999; Joarder \& Narayanan 2000; TerraHomem, Erdélyi \& Ballai 2003) have been examined. One obvious effect of a steady flow is to introduce a Doppler shift in the dispersion curves, but there are also more subtle effects such as the occurrence of negative energy waves.

Finally, there is the question of the generation of the various modes of oscillation of a coronal flux tube. This is an important topic deserving of greater attention, but only a few aspects have so far been discussed (e.g., Roberts et al. 1983, 1984; Aschwanden et al. 2002; Wang 2006; Nakariakov 2007; Terradas, Andries \& Goossens 2007) and much more is needed in the future.

We turn now to a more detailed consideration of two of the above complexities, namely longitudinal structuring and the cause of damping by resonant absorption.

\subsection{Longitudinal structuring: the period ratio $P_{1} / 2 P_{2}$}

Prompted by the observations of Verwichte et al. (2004), Andries et al. (2005a, b; see also Goossens, Andries \& Arregui 2006) have suggested a new tool for coronal seismology that promises to shed light on the nature of longitudinal structuring along a loop, particularly of the plasma density. The idea rests on an interpretation of the harmonics of the loop. Currently, there are only a few observations of such harmonics, but the work of Verwichte et al. (2004) and more recently Van Doorsselaere et al. (2007) provides us with some good candidates for such higher harmonics being present together with the fundamental.

In an elastic string, the ratio of the period $P_{1}$ of the fundamental mode and its first harmonic of period $P_{2}$ is exactly two, so the period ratio $P_{1} / 2 P_{2}$ is unity. However, if there is any cause of dispersion in the wave - as arises naturally in a magnetic flux tube, by virtue of its finite cross-section - then the period ratio departs from unity. McEwan et al. (2006) carried out a systematic assessment of various effects that cause a period shift, including the natural dispersion of the waves, and concluded that longitudinal structuring is the dominant effect - a result implicitly assumed in the studies by Andries et al. $(2005 \mathrm{a}, \mathrm{b})$. Consequently, any shift in the period ratio contains information about the longitudinal structuring of the plasma. Other applications involving the ratio of the fundamental mode of oscillation to higher harmonics are discussed in Erdélyi \& Verth (2006) and Verth et al. (2007), who argue that a knowledge of the behaviour of the eigenfunctions can be used to obtain information about structuring.

The shift in the period ratio may in principle be studied in detail for any mode of a coronal flux tube, but it is often convenient to concentrate on the case of a thin tube. The thin tube approximation has been widely applied to photospheric flux tubes stratified by gravity (Ryutov \& Ryutova 1976; Roberts \& Webb 1978; Spruit 1981) but recently its use in coronal structures has been demonstrated by Dymova \& Ruderman (2005, 2006a, b) who have shown that in the thin tube limit for non-axisymmetrical kink $(m=1)$ oscillations the radial velocity $v_{r} \mathrm{e}^{i \omega t}$ in a $\beta=0$ plasma satisfies a wave equation of the form

$$
\frac{d^{2} v_{r}}{d z^{2}}+\frac{\omega^{2}}{c_{\mathrm{k}}^{2}(z)} v_{r}=0,
$$

where now the kink speed $c_{k}(z)$, defined in (2.9), varies along the tube because of longitudinal structuring in the equilibrium densities inside and outside the tube. It is interesting to note that wave equations of the general form (4.1), but with the propagation speed modified accordingly, arise also for torsional Alfvén waves (with the speed $c_{k}$ replaced by the Alfvén speed; see Zaqarashvili \& Murawski 2007) and slow modes (with $c_{k}$ replaced by $c_{t}$; see Roberts 2006; Díaz \& Roberts 2006). 
Equation (4.1) is amenable to a variety of analytical approaches. McEwan, Díaz \& Roberts (2007) consider the solution of (4.1) for a profile of $c_{\mathrm{k}}^{2}(z)$ that is exponential in form. A variety of other profiles may also be treated (see Dymova \& Ruderman 2005, 2006a, b; Donnelly, Díaz \& Roberts 2007; Erdélyi \& Verth 2007; McEwan et al. 2007; Verth et al. 2007). We suppose that the plasma densities $\rho_{0}(z)$ inside and $\rho_{e}(z)$ outside the loop are exponentials with the same scale height $\Lambda_{c}$, and assume for simplicity that there is equilibrium symmetry about the loop apex. We take the loop to be of length $2 L$ with the loop apex at $z=0$, and then consider the region $0 \leqslant z \leqslant L$ within which the plasma densities $\rho_{0}(z)$ inside and $\rho_{e}(z)$ are

$$
\rho_{0}(z)=\rho_{0}(0) \mathrm{e}^{z / \Lambda_{c}}, \quad \rho_{e}(z)=\rho_{e}(0) \mathrm{e}^{z / \Lambda_{c}} .
$$

Then (4.1) has solution

$$
v_{r}(z)=A J_{0}\left(\lambda \mathrm{e}^{z / 2 \Lambda_{c}}\right)+B Y_{0}\left(\lambda \mathrm{e}^{z / 2 \Lambda_{c}}\right),
$$

where $J_{0}$ and $Y_{0}$ denote the zeroth order Bessel functions and $\lambda=2 \Lambda_{c} \omega / c_{\mathrm{k}}(0)$.

Consider the standing modes of oscillation. Line-tying at the photospheric/chromospheric base requires that $v_{r}=0$ at $z=L$. Conditions at the loop apex $z=0$ then determine the particular modes of oscillation. For even modes the radial velocity $v_{r}$ is symmetric about the loop apex, so that $d v_{r} / d z=0$ at $z=0$; the oscillation has a maximum or minimum at the loop apex. The dispersion relation for the even modes then follows from (4.3) and these boundary conditions:

$$
J_{0}^{\prime}(\lambda) Y_{0}\left(\lambda \mathrm{e}^{L / 2 \Lambda_{c}}\right)-Y_{0}^{\prime}(\lambda) J_{0}\left(\lambda \mathrm{e}^{L / 2 \Lambda_{c}}\right)=0 .
$$

Here a dash (') denotes the derivative of a Bessel function: e. g. $J_{0}^{\prime}(\lambda)$ denotes $d J_{0}(z) / d z$ calculated at $z=\lambda$.

Similarly, the odd modes have a radial motion $v_{r}$ that has a node at the loop apex $z=0 ;$ these modes satisfy the dispersion relation

$$
J_{0}(\lambda) Y_{0}\left(\lambda \mathrm{e}^{L / 2 \Lambda_{c}}\right)-Y_{0}(\lambda) J_{0}\left(\lambda \mathrm{e}^{L / 2 \Lambda_{c}}\right)=0
$$

McEwan et al. (2007) used the dispersion relations (4.4) and (4.5) to obtain approximate expressions for the periods $P_{1}$ and $P_{2}$ of the fundamental and its first harmonic of the loop (of length $2 L$ ) as a whole, valid for weak or moderate longitudinal structuring. Specifically, $P_{1}$ is given approximately by

$$
\frac{P_{\text {kink }}}{P_{1}}=1-\left(\frac{\pi}{4}-\frac{1}{\pi}\right)\left(\frac{L}{\pi \Lambda_{c}}\right)+\left(\frac{\pi^{2}}{48}-\frac{1}{8}-\frac{1}{\pi^{2}}\right)\left(\frac{L}{\pi \Lambda_{c}}\right)^{2}, \quad L \ll \pi \Lambda_{c},
$$

where $P_{\text {kink }}$ is the fundamental period of a fast kink wave in a loop with no longitudinal structuring $\left(\Lambda_{c} \rightarrow \infty\right)$,

$$
P_{\mathrm{kink}}=\frac{4 L}{c_{\mathrm{k}}(0)}
$$

Similarly, $P_{2}$ is determined by

$$
\frac{P_{\text {kink }}}{2 P_{2}}=1-\frac{\pi}{4}\left(\frac{L}{\pi \Lambda_{c}}\right)+\left(\frac{\pi^{2}}{48}-\frac{1}{32}\right)\left(\frac{L}{\pi \Lambda_{c}}\right)^{2}, \quad L \ll \pi \Lambda_{c} .
$$

In the limit of a loop with no longitudinal structuring $\left(\Lambda_{c} \rightarrow \infty\right)$, (4.6) and (4.8) give $P_{1}=2 P_{2}=P_{\text {kink }}$. 
Expressions (4.6) and (4.8) determine the periods $P_{1}$ and $P_{2}$ in terms of loop length $2 L$ and the kink mode period $P_{\text {kink }}$, for a loop that is exponentially structured in density (with profiles given by (4.2)). However, by forming the ratio of the two periods we may eliminate the timescale $P_{\text {kink }}$ :

$$
\frac{P_{1}}{2 P_{2}}=1-\frac{1}{\pi^{2}} \frac{L}{\Lambda_{c}}+\left(\frac{2}{\pi^{4}}-\frac{5}{32 \pi^{2}}\right)\left(\frac{L}{\Lambda_{c}}\right)^{2}, \quad L \ll \pi \Lambda_{c} .
$$

This formula relates the period ratio $P_{1} / 2 P_{2}$ to the ratio $L / \pi \Lambda_{c}$, and so may be used to deduce $\Lambda_{c}$ from a knowledge of the period ratio and loop length $2 L$ (given the assumed model of the density profile).

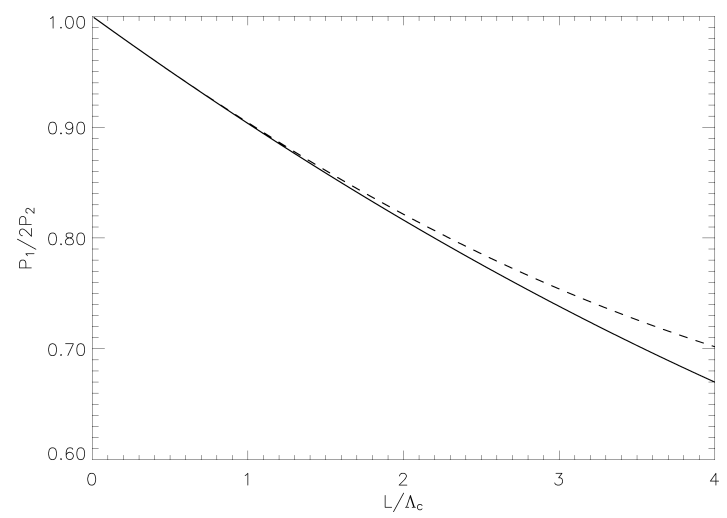

Figure 2. The period ratio $P_{1} / 2 P_{2}$ for a thin tube with exponential longitudinal structuring. The dashed curve corresponds to the numerically determined solution of the dispersion relations (4.4) and (4.5); the solid curve corresponds to the analytical approximation (4.9). (After McEwan, Díaz \& Roberts (2007).)

Figure 2 shows the variation of $P_{1} / 2 P_{2}$ with $L / \Lambda_{c}$ as determined by (4.9). Also shown are the results from an exact solution of the full dispersion relations (4.4) and (4.5). The agreement is excellent.

The case of a general profile may be treated by a variational approach. Multiplying (4.1) by $v_{r}$ and carrying out an integration by parts leads to (McEwan et al. 2007)

$$
\omega^{2}=\frac{I}{J}
$$

where

$$
I=\int_{0}^{L}\left(\frac{d v_{r}}{d z}\right)^{2} d z, \quad J=\int_{0}^{L} \frac{1}{c_{\mathrm{k}}^{2}(z)} v_{r}^{2} d z .
$$

The boundary conditions that $v_{r}=0$ at the base of the loop $(z=L)$ and that $v_{r} d v_{r} / d z=$ 0 at the loop apex, which hold for the even or odd modes, have been applied in obtaining (4.10). The integrals $I$ and $J$ may be evaluated for any given profile for $c_{k}^{2}(z)$ once $v_{r}(z)$ is specified. Equation (4.10) provides a variational formulation of our eigenvalue problem used in the Rayleigh-Ritz procedure. McEwan et al. (2007) exploit this approach by choosing simple trial functions for $v_{r}(z)$ which satisfy the boundary conditions at the loop apex and base:

$$
v_{r}(z)= \begin{cases}\cos \left(\frac{\pi z}{2 L}\right), & \text { for } P_{1} \\ \sin \left(\frac{\pi z}{L}\right), & \text { for } P_{2}\end{cases}
$$


These trial functions are chosen to give the appropriate behaviour for $v_{r}(z)$ that accords with the fundamental mode of period $P_{1}$ and its first harmonic of period $P_{2}$. Knowledge of the exact eigenfunctions $v_{r}(z)$ leads to an exact determination of $\omega^{2}$, but even an approximation to those functions leads to a good approximation to $\omega^{2}$ through (4.10). By evaluating the integrals $I$ and $J,(4.10)$ determines the frequency $\omega$ (and so the periods $P_{1}$ and $\left.P_{2}\right)$ for any choice of profile $c_{\mathrm{k}}^{2}(z)$. An alternative, complementary, approach is to use the WKB approximation (see McEwan et al. 2007).

\subsection{Damping}

The almost ubiquitous presence of damping in standing loop oscillations observed by TRACE and SoHO/SUMER, be they fast kink modes or slow modes, raises the question of the cause of this decay, though the freely propagating wave observed by $S E C I S$ does not share this feature. It may well be that more than one mechanism is responsible. Roberts (2000) reviewed a number of possibilities for explaining the damping of kink mode oscillations.

The damping of longitudinal slow mode oscillations seems to have been satisfactorily explained as due to thermal conduction, efficient in the high temperatures $(6-10 \mathrm{MK})$ associated with oscillating SUMER loops, or viscous effects (Ofman \& Wang 2002; De Moortel \& Hood 2003, 2004; Mendoza-Briceño, Erdélyi \& Sigalotti 2004; Taroyan et al. 2007).

The damping of fast kink oscillations appears to more problematic, though a good case has been made for resonant absorption. Indeed, Ruderman \& Roberts (2002) showed that the Nakariakov et al. (1999) observation of a decaying kink oscillation could be explained as resonant absorption acting in a thin layer of width $l=0.23 a$ forming a transition layer between the inside of a loop and its environment. This result was very shortly thereafter extended by Goossens, Andries \& Aschwanden (2002) who examined eleven cases selected in Ofman \& Aschwanden (2002), concluding that resonant absorption is able to reproduce the observed decay for a range of $l / a$ extending from 0.16 to 0.46 . The effect seems to be of wide generally. The fact that the upper estimate $l / a=0.46$ is somewhat outside the assumed thin layer ( $l / a$ small) explored analytically by Ruderman \& Roberts (2002) has led to numerical investigations of damping (Van Doorsselaere et al. 2004b; Arregui et al. 2007a). The general conclusion is that results similar to the thin layer model pertain if one allows a suitable weighting in a geometrical factor $C$ (see below). It is interesting to note that Hollweg \& Yang (1988) predicted well in advance of the first observations that kink oscillations of loops would be strongly damped by resonant absorption.

The effect of resonant absorption is to produce a decay in a standing kink oscillation of period $P$, as the kink oscillations transfer energy into azimuthal motions. In the thin layer case, the decay proceeds on a timescale $\tau$ given by (Ruderman \& Roberts 2002; see also Hollweg \& Yang 1988; Goossens, Hollweg \& Sakarai 1992)

$$
\tau=C\left(\frac{a}{l}\right)\left(\frac{\rho_{0}+\rho_{e}}{\rho_{0}-\rho_{e}}\right) P,
$$

where the factor $C$ depends upon the geometrical detail of the radial density profile $\rho_{0}(r)$. For a profile $\rho_{0}(r)$ which changes smoothly and monotonically from a uniform density $\rho_{0}$ in $0 \leqslant r<a-l$ to a uniform value $\rho_{e}$ in $r \geqslant a$ across a thin layer of width $l(\ll a)$ on the tube boundary, $C$ is given by

$$
C=\left\{\begin{array}{cl}
2 / \pi, & \text { sinusoidal profile } \\
4 / \pi^{2}, & \text { linear profile }
\end{array}\right.
$$


Thus, the precise nature of the thin density layer may modify the resulting decay time $\tau$ by a factor $C=0.637$ or $C=0.405$. In a wide layer, numerical investigation shows that $C$ may change even further (Aschwanden et al. 2003; Van Doorsselaere et al. 2004b; see also Goossens et al. 2005; Arregui et al. 2007a), but essentially the effect is the same.

Alternatives to resonant absorption have also been discussed, ranging from phase mixing (Ofman \& Aschwanden 2002), to lateral radiation because of the geometrical curvature of loops or arcades (Brady \& Arber 2005; Verwichte, Foullon \& Nakariakov 2006a-c; Díaz, Zaqarashvili \& Roberts 2006; Díaz 2006), to the simple passage of a disturbing wave generating an oscillatory wake (Uralov 2003; Terradas, Oliver \& Ballester 2005).

\section{Conclusions}

Whilst progress in the new field of coronal seismology has been impressive, showing the power of combining the theoretical tools of mathematical modelling (analytical and numerical) with high accuracy observations, there are of course a number of problems remaining. Indeed, many of the problems that present themselves now were already apparent and discussed in Roberts (2000). There remains the issue of damping: what mechanism (or mechanisms) is responsible for the observed decay of oscillations? The case for resonant absorption is a strong one, but not yet secured; more observations are needed to test these ideas.

Numerical simulations - in the main not discussed here - will undoubtedly play a key role in future developments, as we seek to understand better the complexities that reality adds to our simple models. Future progress will undoubtedly involve a full comparison between observations, analytical models and simulations. That way we may hope to understand better the plasmas that manifest wave phenomena.

\section{Acknowledgements}

I am grateful to Professors Robertus Erdélyi and César Mendoza-Briceño for affording me the opportunity to address this topic in the pleasant location of Isla de Margarita.

\section{References}

Abramowitz, A., \& Stegun, I. A. 1965, Handbook of Mathematical Functions, Dover Publications, New York

Andries, J., Goossens, M., Hollweg, J. V., Arregui, I., \& Van Doorsselaere, T. 2005a, A\&̛A 430, 1109

Andries, J., Arregui, I., \& Goossens, M. 2005b, ApJ 624, L57

Arregui, I., Andries, J., Van Doorsselaere, T., Goossens, M., \& Poedts, S. 2007a, A 6 A 463, 333

Arregui, I., Terradas, J., Oliver, R., \& Ballester, J. L. 2007b, A\&A 466, 1145

Aschwanden, M. J. 1987, Solar Phys. 111, 113

Aschwanden, M. J. 2004, Physics of the Solar Corona, Springer

Aschwanden, M. J., Fletcher, L., Schrijver, C. J. \& Alexander, D. 1999, ApJ 520, 880

Aschwanden, M. J., De Pontieu, B., Schrijver, C. J. \& Title, A. 2002, Solar Phys. 206, 99

Aschwanden, M. J., Nightingale, R. W., Andries, J., Goossens, M. \& Van Doorsselaere, T. 2003, ApJ 598, 1375

Aschwanden, M. J., Nakariakov, V. M., \& Melnikov, V. F. 2004, ApJ 600, 458

Ballai, I., Erdélyi, R., \& Pintér, B. 2005, ApJ 633, L145

Ballester, J. L. 2003, in R. Erdélyi, K. Petrovay, B. Roberts and M. Aschwanden (eds.), Turbulence, Waves and Instabilities in the Solar Plasma, NATO Advanced Research Workshop, Budapest, p. 193

Banerjee, D., Erdélyi, R., Oliver, R., \& O'Shea, E. 2007, Solar Phys. 246, 3

Bennett, K., Roberts, B., \& Narain, U. 1999, Solar Phys. 185, 41 
Berghmans, D., \& Clette, F. 1999, Solar Phys. 186, 207

Brady, C. S. \& Arber, T. D. 2005, A\&A 438, 733

Cally, P. S. 1986, Solar Phys. 103, 27

Carter, B. K., \& Erdélyi, R. 2007, A\& A 475, 323

DeForest, C. E., \& Gurman, J. B. 1998, ApJ 501, L217

Defouw, R. J. 1976, ApJ 209, 266

De Moortel, I. 2005, Phil. Trans. R. Soc. A 363, 2743

De Moortel, I., \& Hood, A. W. 2000, A\&A 363, 269

De Moortel, I., \& Hood, A. W. 2003, A\& $A$ 408, 755

De Moortel, I., \& Hood, A. W. 2004, A\& A 415, 705

De Moortel, I., Hood, A. W., Ireland, J., \& Walsh, R. W. 2002a, Solar Phys. 209, 89

De Moortel, I., Ireland, J., Hood, A. W., \& Walsh, R. W. 2002b, A\&SA 387, L13

De Moortel, I., Ireland, J., \& Walsh, R. W. 2000, A\&A 355, L23

De Moortel, I., Ireland, J., Walsh, R. W., \& Hood, A. W. 2002c, Solar Phys. 209, 61

Díaz, A. J. 2004, PhD thesis, Universitat de les Illes Balears, Palma de Mallorca

Díaz, A.J. 2006, A\&A 456, 737

Díaz, A. J., Oliver, R., \& Ballester, J. L. 2005, A\&A 440, 1167

Díaz, A. J., \& Roberts, B. 2006, A\& A 458, 975

Díaz, A. J., Zaqarashvili, T., \& Roberts, B. 2006, A\&A 455, 709

Donnelly, G. R., Díaz, A.J. \& Roberts, B. 2007, A\& $A$ 471, 999

Dungey, J. W., \& Loughhead, R. E. 1954, Australian J. Phys. 7, 5

Dymova, M. V., \& Ruderman, M. S. 2005, Solar Phys. 229, 79

Dymova, M. V., \& Ruderman, M. S. 2006a, A\&SA, 457, 1059

Dymova, M. V., \& Ruderman, M. S. 2006b, A\&A, 459, 241

Edwin, P. M., \& Roberts, B. 1982, Solar Phys. 76, 239

Edwin, P. M., \& Roberts, B. 1983, Solar Phys. 88, 179

Erdélyi, R., \& Carter, B. K. 2006, AछA, 455, 361

Erdélyi, R. \& Fedun, V. 2006, Solar Phys. 238, 41

Erdélyi, R. \& Fedun, V. 2007, Solar Phys. 246, 101

Erdélyi, R., \& Verth, G. 2007, AESA, 462, 743

Foullon, C., Verwichte, E., Nakariakov, V. M., \& Fletcher, L. 2005, A\&\&A 440, L59

Goossens, M. 1991, in: E. R. Priest \& A. W. Hood (eds.), Advances in Solar System Magnetohydrodynamics, Cambridge University Press, p. 137

Goossens, M., Andries, J., \& Aschwanden, M. J. 2002, A $\mathscr{J} A$ 394, L39

Goossens, M., Andries, J., Arregui, I., Van Doorsselaere \& Poedts, S. 2005, in: E. M. de Gouveia Dal Pino et al. (eds.), American Inst. Phys. CP784, Magnetic Fields in the Universe: From Laboratory and Stars to Primordial Structures, p. 114

Goossens, M., Andries, J., \& Arregui, I. 2006, Phil. Trans. R. Soc. A 364, 433

Goossens, M., Hollweg, J. V., \& Sakurai, T. 1992, Solar Phys. 138, 233

Gruszecki, M, Murawski, K., Selwa, M., \& Ofman, L. 2006, A\& A 460, 887

Hollweg, J. V., \& Yang, G. 1988, J. Geophysical Res. 93, 5423

Joarder, P. S., Nakariakov, V. M., \& Roberts, B. 1997, Solar Phys. 176, 285

Joarder, P. S., \& Narayanan, A. S. 2000, A\&A 359, 1211

Katsiyannis, A. C., Williams, D. R., McAteer, R. T. J., Gallagher, P. T., Keenan, F. P., \& Murtagh, F. 2003, A\&A 406, 709

Kliem, B., Dammasch, I. E., Curdt, W., \& Wilhelm, K. 2002, ApJ 568, L61

Kopylova, Y. G., Stepanov, A. V. \& Tsap, Y. T. 2002, Astronomy Letters 28, 783

Kukhianidze, V., Zaqarashvili, T. V., \& Khutsishvili, E. 2006 A $\& A$ 449, L35

Luna, M, Terradas, J., Oliver, R., \& Ballester, J. L. 2006, A\&A 457, 1071

Mariska, J. T. 2005, ApJ 620, L67

Mariska, J. T. 2006, ApJ 639, 484

Mathioudakis, M., Bloomfield, D. S., Jess, D. B., Dhillon, V. S., \& Marsh, T. R. 2006, A\& A 458,921

McEwan, M. P., Donnelly, G. R., Díaz, A. J., \& Roberts, B. 2006, A\&A 460, 893 
McEwan, M.P., Díaz, A. J., \& Roberts, B. 2007, $A \mathscr{E} A$ in press

McKenzie, J. F. 1970, Geophys. Res., Space Phys. 75, 5331

Mendoza-Briceño, C. A., Erdélyi, R., \& Sigalotti, L. Di G. 2004, ApJ 605, 493

Mikhalyaev, B. B. 2005, Astronomy Letters 31, 406

Mitra-Kraev, U., Harra, L. K., Williams, D. R., \& Kraev, E. 2005, A\&̈A 436, 1041

Nakariakov, V. M. 2007, Advances in Space Research 39, 1804

Nakariakov V. M., Arber, T. D., Ault, C. E., Katsiyannis, A. C., Williams, D. R., \& Keenan, F. P. 2004, MNRAS 349, 705

Nakariakov, V. M., Melnikov, V. F., \& Reznikova, V. E. 2003, A\&A 412, L7

Nakariakov, V. M., \& Ofman, L. 2001, A\& A 372, L53

Nakariakov, V. M., Ofman, L., \& Arber, T. D. 2000, A\&A 353, 741

Nakariakov, V. M., Ofman, L., DeLuca, E. E., Roberts, B., \& Davila, J. M. 1999, Science 285, 862

Nakariakov, V. M., Pascoe, D. J., \& Arber, T. D. 2005, Space Science Reviews 121, 115

Nakariakov, V. M., \& Roberts, B. 1995a, Solar Phys. 159, 213

Nakariakov, V. M., \& Roberts, B. 1995b, Solar Phys. 159, 399

Nakariakov, V. M., \& Verwichte, E. 2005, Living Reviews Solar Phys. 2, 3

Narayanan, A. S. 1991, Plasma Phys. Control. Fusion 33, 333

Ofman, L. 2005, Advances in Space Research 36, 1572

Ofman, L., \& Aschwanden, M. J. 2002, ApJ 576, L153

Ofman, L., Romoli, M., Poletto, G., Noci, G., \& Kohl, J. L. 1997, ApJ 491, L111

Ofman, L., Nakariakov, V. M., \& DeForest, C. E. 1999, ApJ 514, 441

Ofman, L., \& Wang, T. J. 2002, ApJ 580, L85

Oliver, R., \& Ballester, J. L. 2002, Solar Phys. 206, 45

Parker, E. N. 1979, Cosmical Magnetic Fields, Clarendon Press, Oxford, §8.10

Pascoe, D. J., Nakariakov, V. M., \& Arber, T. D. 2007a, A\&3A 461, 1149

Pascoe, D. J., Nakariakov, V. M., \& Arber, T. D. 2007b, Solar Phys. preprint

Pekeris, C. L. 1948, Geol. Soc. Am. Memoir 27

Reznikova, V. E., Melnikov, V. F., Nakariakov, V. M., \& Shibasaki, K. 2006, in: N. H. Solomos (ed.) Recent Advances in Astronomy and Astrophysics, CP848 (Amer. Inst.Phys.), p. 133

Robbrecht, E., Verwichte, E., Berghmans, D., Hochedez, J. F., Poedts, S. \& Nakariakov, V. M. 2001, A\&A 370, 591

Roberts, B. 1981a, Solar Phys. 69, 27

Roberts, B. 1981b, Solar Phys. 69, 39

Roberts, B. 2000, Solar Phys. 193, 139

Roberts, B. 2002, in: A. Wilson (ed.), Solar Variability: From Core to Outer Frontiers, ESA SP-506, p. 481

Roberts, B. 2004, in: R. Erdélyi, J. L. Ballester \& B. Fleck (eds.), SoHO 13, Waves, Oscillations and Small Scale Transient Events in the Solar Atmosphere: A Joint View From SoHO and TRACE, ESA SP-547, p. 1

Roberts, B. 2006, Phil. Trans. R. Soc. A 364, 447

Roberts, B., Edwin, P. M., \& Benz, A. O. 1983, Nature 305, 688

Roberts, B., Edwin, P.M., \& Benz, A.O. 1984, ApJ 279, 857

Roberts, B., \& Nakariakov, V.M. 2003, in: R. Erdélyi, K. Petrovay, B. Roberts \& M. J. Aschwanden (eds.), Turbulence, Waves, and Instabilities in the Solar Plasma, Nato Advanced Research Workshop, NATO ASI Series, (Kluwer Academic Publishers), p. 167

Roberts, B., \& Webb, A. R. 1978, Solar Phys. 56, 5

Roberts, B., \& Webb, A. R. 1979, Solar Phys. 59, 249

Ruderman, M. S. 2003, A\&A 409, 287

Ruderman, M. S. 2007, Solar Phys. 246, 119

Ruderman, M. S., \& Roberts, B. 2002, ApJ 577, 475

Ryutov, D. D., \& Ryutova, M. P. 1976, Sov. Phys. JETP 43, 491

Schrijver, C. J., Title, A. M., Berger, T. E.., et al. 1999, Solar Phys. 187, 261

Schrijver, C. J., Aschwanden, M. J., \& Title, A. M. 2002, Solar Phys. 206, 69

Singh, K. A. P \& Dwivedi, B. N. 2007, New Astronomy 12, 479 
Solov'ev, A. A., Mikhalyaev, B. B., \& Kiritchek, E. A. 2002a, Plasma Physics Reports 28, 699

Solov'ev, A. A., Mikhalyaev, B. B., \& Kiritchek, E. A. 2002b, Plasma Physics Reports 29, 1049

Somasundaram, K., Venkatraman, S., \& Sengottuvel, M. P. 1999, Plasma Phys. Control. Fusion 41,1421

Spruit, H. C. 1981, A\&A 98, 155

Spruit, H. C. 1982, Solar Phys. 75, 3

Taroyan, Y., Erdélyi, R., Wang, T. J., \& Bradshaw, S. J. 2007, ApJ 659, L173

Terradas, J., Andries, J. \& Goossens, M. 2007, A\&SA 469, 1135

Terradas, J., Oliver, R. \& Ballester, J. L. 2005, ApJ 618, L149

Terra-Homem, M., Erdélyi, R., \& Ballai, I. 2003, Solar Phys. 217, 199

Tirry, W. J., Cadez, W. M., Erdélyi, R., \& Goossens, M. 1998, A\&A 332, 786

Uberoi, C. \& Somasundaram, K. 1980, Plasma Phys. 22, 747

Uchida, Y. 1968, Solar Phys. 4, 30

Uchida, Y. 1970, Publ. Astron. Soc. Japan 22, 341

Uchida, Y. 1973, Solar Phys. 28, 495

Uchida, Y. 1974, Solar Phys. 39, 431

Uralov, A. M. 2003, Astron.Letters 29, 486

Van Doorsselaere, T., Debosscher, A., Andries, J., \& Poedts, S. 2004a, A $\& A$ 424, 1065

Van Doorsselaere, T., Andries, J., Poedts, S., \& Goossens, M. 2004b, ApJ 606, 1223

Van Doorsselaere, T., Nakariakov, V. M., Verwichte, E. 2007, A $\mathscr{U} A$ 473, 959

Verth, G., Van Doorsselaere, T., Erdélyi, R., \& Goossens, M. 2007, A $\& A$ 475, 341

Verwichte, E., Foullon, C., \& Nakariakov, V. M. 2006a, A\&A 446, 1139

Verwichte, E., Foullon, C., \& Nakariakov, V. M. 2006b, A\& $A$ 449, 769

Verwichte, E., Foullon, C., \& Nakariakov, V. M. 2006c, A\&\&A 452, 615

Verwichte, E., Nakariakov, V. M., \& Cooper, F. C. 2005, A\&A 4 430, L65

Verwichte, E., Nakariakov, V. M., Ofman, L., \& DeLuca, E. E. 2004, Solar Phys. 233, 77

Wang, T. J. 2004, in: R. Erdélyi, J. L. Ballester \& B. Fleck (eds.), SoHO 13, Waves, Oscillations and Small Scale Transient Events in the Solar Atmosphere: A Joint View From SoHO and TRACE, ESA SP-547, p. 417

Wang, T. J. 2006, in: D. Danesy (ed.) Chromospheric and Coronal Magnetic Fields, ESA SP-596

Wang, T. J., Innes, D. E., \& Qiu, J. 2007, ApJ 656, 598

Wang, T. J., \& Solanki, S. K. 2004, A\& A 421, L33

Wang, T. J., Solanki, S. K., Curdt, W., Innes, D. E., \& Dammasch, I. E. 2002, ApJ 574, L101

Wang, T. J., Solanki, S. K., Curdt, W., Innes, D. E., Dammasch, I. E., \& Kliem, B. 2003a, A\&\&A 406, 1105

Wang, T. J., Solanki, S. K., Innes, D. E., Curdt, W., \& Marsch, E. 2003b, A $\& A$ 402, L17

Williams, D. R., Phillips, K. J. H., Rudawy, P., Mathioudakis, M., Gallagher, P. T., O'Shea, E., Keenan, F. P., Read, P., \& Rompolt, B. 2001, MNRAS 326, 428

Williams, D. R., Mathioudakis, M., Gallagher, P. T., Phillips, K. J. H., McAteer, R. T. J., Keenan, F. P., Rudawy, P., \& Katsiyannis, A. C. 2002a, MNRAS 336, 747

Williams, D. R., Mathioudakis, M., Gallagher, P. T., Phillips, K. J. H., McAteer, R. T. J., Keenan, F. P. \& Katsiyannis, A. C. 2002b, in: Proc. SOLMAG, IAU Colloq. 188, Santorini (ESA SP-505), p. 615

Zaitsev, V. V. \& Stepanov, A. V. 1975, Issled. Geomagn. Aeron. Fiz. Solntsa 37, 3

Zaqarashvili, T. V. 2003, A $\& A$ 390, L15

Zaqarashvili, T. V., Khutsishvili, E., Kukhianidze, V., \& Ramishvili, G. 2007 A $\& A$ 474, 627

Zaqarashvili, T. V., \& Murawski, K. 2007, A\&A 470, 353 